\title{
Erratum to: Magnitude and Trophic Fate of Black Needlerush (Juncus Roemerianus) Productivity: Does Nutrient Addition Matter?
}

\author{
Amy Hunter • Just Cebrian • Jason P. Stutes • \\ David Patterson • Bart Christiaen • Celine Lafabrie • \\ Josh Goff
}

Published online: 26 January 2015

(C) Society of Wetland Scientists 2015

\section{Erratum to: Wetlands}

DOI 10.1007/s13157-014-0611-5

Table 2 in the paper Magnitude and Trophic Fate of Black Needlerush (Juncus Roemerianus) Productivity: Does Nutrient Addition Matter? is missing the heading for the variables "Rhizome Biomass", "Root Biomass", "Carbon to Nitrogen ratio in live leaf biomass" and "Carbon to Nitrogen ratio in dead leaf biomass". This head-

ing should be located following the "Within Subjects" "Error" row for the variables "Porewater $\mathrm{N}$ concentration", "Porewater P concentration", "Aboveground live biomass" and "Aboveground dead biomass". The "Within Subjects" "Error" row for the variables "Rhizome Biomass", "Root Biomass", "Carbon to Nitrogen ratio in live leaf biomass" and "Carbon to Nitrogen ratio in dead leaf biomass" is also missing. We now provide the correct version of Table 2 .

The online version of the original article can be found at http://dx.doi.org/ 10.1007/s13157-014-0611-5.

\section{A. Hunter}

Department of Biological Sciences, University of Alabama,

Tuscaloosa, AL 35487, USA

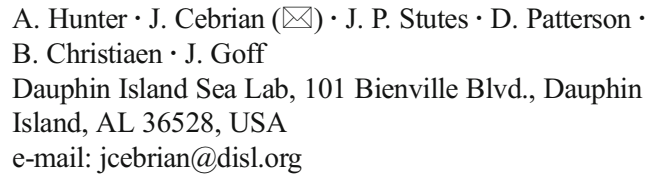


Table 2 Results of repeated-measures ANOVA

\begin{tabular}{|c|c|c|c|c|c|c|c|c|c|c|c|c|}
\hline \multirow[b]{2}{*}{ Source of variation } & \multicolumn{3}{|c|}{ Porewater $\mathrm{N}$ concentration } & \multicolumn{3}{|c|}{ Porewater P concentration } & \multicolumn{3}{|c|}{ Aboveground live biomass } & \multicolumn{3}{|c|}{ Aboveground dead biomas } \\
\hline & $\mathrm{df}$ & $\mathrm{F}$ & $p$ & df & $\mathrm{F}$ & $p$ & $\mathrm{df}$ & $\mathrm{F}$ & $p$ & df & $\mathrm{F}$ & $p$ \\
\hline \multicolumn{13}{|l|}{ Between subjects } \\
\hline Site & 1 & 2.3 & 0.16 & 1 & 15.5 & $\leq 0.05$ & 1 & 0.9 & 0.36 & 1 & 1.6 & 0.23 \\
\hline Treatment & 1 & 9.7 & $\leq 0.05$ & 1 & 8.3 & $\leq 0.05$ & 1 & 0.0 & 0.95 & 1 & 0.5 & 0.51 \\
\hline Site $\times$ treatment & 1 & 0.3 & 0.60 & 1 & 0.0 & 0.95 & 1 & 0.3 & 0.60 & 1 & 0.4 & 0.53 \\
\hline Error & 11 & & & 11 & & & 10 & & & 10 & & \\
\hline \multicolumn{13}{|l|}{ Within subjects } \\
\hline Time & 14 & 17.8 & $\leq 0.05$ & 14 & 10.6 & $\leq 0.05$ & 6 & 6.5 & $\leq 0.05$ & 6 & 2.7 & $\leq 0.05$ \\
\hline Time $\times$ site & 14 & 1.5 & 0.10 & 14 & 1.5 & 0.11 & 6 & 2.7 & $\leq 0.05$ & 6 & 1.8 & 0.12 \\
\hline Time $\times$ treatment & 14 & 4.3 & $\leq 0.05$ & 14 & 2.8 & $\leq 0.05$ & 6 & 0.8 & 0.57 & 6 & 0.8 & 0.57 \\
\hline Time $\times$ site $\times$ treatment & 14 & 0.6 & 0.84 & 14 & 0.8 & 0.64 & 6 & 1.2 & 0.33 & 6 & 1.1 & 0.36 \\
\hline \multirow[t]{2}{*}{ Error } & \multicolumn{3}{|c|}{154} & \multicolumn{3}{|l|}{154} & \multicolumn{3}{|l|}{60} & \multicolumn{3}{|l|}{60} \\
\hline & \multicolumn{3}{|c|}{ Rhizome biomass } & \multicolumn{3}{|c|}{ Root biomass } & \multicolumn{3}{|c|}{$\begin{array}{l}\text { Carbon to Nitrogen ratio in } \\
\text { live leaf biomass }\end{array}$} & \multicolumn{3}{|c|}{$\begin{array}{l}\text { Carbon to Nitrogen ratio in } \\
\text { dead leaf biomass }\end{array}$} \\
\hline Source of variation & $\mathrm{df}$ & $\mathrm{F}$ & $p$ & df & F & $p$ & $\mathrm{df}$ & $\mathrm{F}$ & $p$ & $\mathrm{df}$ & $\mathrm{F}$ & $p$ \\
\hline \multicolumn{13}{|l|}{ Between subjects } \\
\hline Site & 1 & 10.7 & $\leq 0.05$ & 1 & 4.0 & 0.07 & 1 & 4.0 & 0.07 & 1 & 9.9 & $\leq 0.05$ \\
\hline Treatment & 1 & 3.9 & 0.07 & 1 & 0.3 & 0.62 & 1 & 3.2 & 0.10 & 1 & 8.1 & $\leq 0.05$ \\
\hline Site $\times$ treatment & 1 & 0.1 & 0.73 & 1 & 0.2 & 0.68 & 1 & 0.3 & 0.60 & 1 & 0.7 & 0.41 \\
\hline Error & 11 & & & 11 & & & 12 & & & 12 & & \\
\hline \multicolumn{13}{|l|}{ Within subjects } \\
\hline Time & 6 & 7.1 & $\leq 0.05$ & 6 & 28.8 & $\leq 0.05$ & 2 & 113.1 & $\leq 0.05$ & 2 & 58.2 & $\leq 0.05$ \\
\hline Time $\times$ site & 6 & 1.6 & 0.16 & 6 & 2.0 & 0.08 & 2 & 5.6 & $\leq 0.05$ & 2 & 1.5 & 0.25 \\
\hline Time $\times$ treatment & 6 & 0.7 & 0.64 & 6 & 1.2 & 0.32 & 2 & 1.3 & 0.28 & 2 & 2.7 & 0.09 \\
\hline Time $\times$ site $\times$ treatment & 6 & 1.2 & 0.34 & 6 & 1.7 & 0.12 & 2 & 1.5 & 0.23 & 2 & 1.6 & 0.22 \\
\hline \multirow[t]{2}{*}{ Error } & 66 & & & 66 & & & 24 & & & 24 & & \\
\hline & \multicolumn{3}{|c|}{$\begin{array}{l}\text { Carbon to Nitrogen ratio in } \\
\text { rhizome biomass }\end{array}$} & $\begin{array}{r}\text { Carb } \\
\text { ro }\end{array}$ & $\begin{array}{l}\text { to } \mathrm{Nit} \\
\text { iomas }\end{array}$ & en ratio in & $\begin{array}{r}\text { Car } \\
1\end{array}$ & $\begin{array}{l}\text { to Pho } \\
\text { eaf bio }\end{array}$ & rus ratio in & & $\begin{array}{l}\text { to } \mathrm{Ph} \\
\text { ead lea }\end{array}$ & $\begin{array}{l}\text { horus ratio } \\
\text { omass }\end{array}$ \\
\hline Source of variation & df & $\mathrm{F}$ & $p$ & df & $\mathrm{F}$ & $p$ & $\mathrm{df}$ & $\mathrm{F}$ & $p$ & $\mathrm{df}$ & $\mathrm{F}$ & $p$ \\
\hline Between subjects & & & & & & & & & & & & \\
\hline Site & 1 & 2.1 & 0.18 & 1 & 37.4 & $\leq 0.05$ & 1 & 13.8 & $\leq 0.05$ & 1 & 15.5 & $\leq 0.05$ \\
\hline Treatment & 1 & 0.0 & 0.85 & 1 & 0.8 & 0.40 & 1 & 1.9 & 0.19 & 1 & 2.0 & 0.18 \\
\hline Site $\times$ treatment & 1 & 1.5 & 0.24 & 1 & 8.2 & $\leq 0.05$ & 1 & 0.0 & 0.91 & 1 & 0.1 & 0.82 \\
\hline Error & 10 & & & 11 & & & 12 & & & 12 & & \\
\hline Within subjects & & & & & & & & & & & & \\
\hline Time & 2 & 13.5 & $\leq 0.05$ & 2 & 33.3 & $\leq 0.05$ & 2 & 14.4 & $\leq 0.05$ & 2 & 3.1 & 0.07 \\
\hline Time $\times$ site & 2 & 1.7 & 0.21 & 2 & 9.7 & $\leq 0.05$ & 2 & 2.2 & 0.13 & 2 & 0.0 & 0.98 \\
\hline Time $\times$ treatment & 2 & 0.9 & 0.43 & 2 & 1.2 & 0.32 & 2 & 0.5 & 0.64 & 2 & 0.1 & 0.93 \\
\hline Time $\times$ site $\times$ treatment & 2 & 0.3 & 0.74 & 2 & 0.1 & 0.90 & 2 & 0.7 & 0.52 & 2 & 0.2 & 0.84 \\
\hline Error & 20 & & & 22 & & & 24 & & & 24 & & \\
\hline & $\begin{array}{r}\mathrm{Car} \\
\mathrm{rl}\end{array}$ & $\begin{array}{l}\text { o Pho } \\
\text { ne bio }\end{array}$ & rus ratio in & $\begin{array}{r}\text { Carb } \\
\text { in }\end{array}$ & $\begin{array}{l}\text { to Phe } \\
\text { t bion }\end{array}$ & horus ratio & Lea & oductiv & & & ory & \\
\hline Source of variation & df & $\mathrm{F}$ & $p$ & df & $\mathrm{F}$ & $p$ & df & F & $p$ & df & $\mathrm{F}$ & $p$ \\
\hline Between subjects & & & & & & & & & & & & \\
\hline Site & 1 & 4.7 & 0.06 & 1 & 0.6 & 0.47 & 1 & 11.5 & $\leq 0.05$ & 1 & 2.1 & 0.17 \\
\hline Treatment & 1 & 1.8 & 0.21 & 1 & 0.9 & 0.38 & 1 & 2.2 & 0.16 & 1 & 0.1 & 0.80 \\
\hline Site $\times$ treatment & 1 & 0.7 & 0.42 & 1 & 0.7 & 0.43 & 1 & 1.1 & 0.32 & 1 & 1.6 & 0.24 \\
\hline Error & 10 & & & 10 & & & 12 & & & 12 & & \\
\hline
\end{tabular}


Table 2 (continued)

\begin{tabular}{|c|c|c|c|c|c|c|c|c|c|c|c|c|}
\hline \multicolumn{13}{|l|}{ Within subjects } \\
\hline Time & 2 & 1.8 & 0.19 & 2 & 0.3 & 0.73 & 5 & 28.4 & $\leq 0.05$ & 5 & 13.6 & $\leq 0.05$ \\
\hline Time $\times$ site & 2 & 0.3 & 0.75 & 2 & 0.3 & 0.74 & 5 & 7.8 & $\leq 0.05$ & 5 & 2.7 & $\leq 0.05$ \\
\hline Time $\times$ treatment & 2 & 0.3 & 0.73 & 2 & 0.6 & 0.57 & 5 & 0.9 & 0.49 & 5 & 1.0 & 0.42 \\
\hline Time $\times$ site $\times$ treatment & 2 & 1.1 & 0.36 & 2 & 0.4 & 0.68 & 5 & 2.0 & 0.10 & 5 & 1.7 & 0.15 \\
\hline Error & 20 & & & 20 & & & 60 & & & 60 & & \\
\hline
\end{tabular}

